NASA-CR-202190

\title{
On the Influence of a Fuel Side Heat-Loss ("Soot") Layer on a Planar Diffusion Flame
}

\author{
INDREK S. WCHMAY* \\ Laboratoire E..H2.C.. Ecole Centrale de Paris. 92295 Chatenav-Halabri. France
}

\begin{abstract}
At model of the response of a diffusion Hame (DF) to an adjacent heat loss or "soot" laver on the fuel side is investigated. The thermal influence of the "soot" or heat-loss layer on the DF occurs through the enthalpy sink it creates. A sink distribution in mixture-fraction space is employed to examine possible DF extinction. It is tound that (i) the enthalpy sink (or "soot" layer) must touch the DF for radiation-induced quenching to occur. and (ii) for fuel-rich conditions extinction is possible oniy for a progressively narrower range of values of the characteristic heat-loss parameter. $\left.\dot{V}_{R}\right\lrcorner Z_{R}$. Various interpretations of the model are discussed. An attempt is made to place this work into the context created by previous experimental and computational studies.
\end{abstract}

\section{NOMENCLATURE}

a constant (see Eq. 13) describing reaction-zone heat loss (Eq. 14)

$a_{R} \quad$ same as $a$ with radiation included

A preexponential factor

$b \quad$ reduced Damköhler number, see Eq. 14

$b_{E} \quad$ reduced Damköhler number at extinction

B E E/3RT , see Eq. 26

C an $O(1)$ constant in the definition of $\eta$, see Eq. 13.ii

$D$ diffusion coefficients for species $i$

$D \quad$ Damköhler number

$D_{R} \quad$ Damköhler number with radiant losses from the reaction zone

DF "diffusion flame"

e $2.718 \ldots$

E activation energy

$h$ enthalpy

$H$ "excess" enthalpy function, generally non-zero

$H_{0} \quad$ same as $H$ with no losses: $H_{o}=0$

HC "hydrocarbon"

$i_{b \lambda}$ blackbody spectral intensity given by Piuncks forminia

- Permanent address: Department of Mechanical Engineering, Michigan State University, East Lansing, MI 48824 USA. $k \quad$ soot surface growth rate

$K$ function defined in Eq. 25 and evaluated in Sec. III.E

$L \quad$ width of the physical domain

$L^{*} \quad$ density-coordinate reduced version of $L$, see Eq. 10

$\mathrm{Le}_{i} \quad$ Lewis number of species $i$

$\dot{m}$ mass generated (destroyed) per unit volume per second (Sec. V)

$N$ "soot" number density, particles/ volume

$N_{R} \quad$ radiation number, see Eq. 2 et seq.

$P \quad$ pressure

$P(x)$ polynomial defined by Eq. 21

$Q_{F} \quad$ heat released by combustion of fuel

$\bar{Q}_{F} \quad$ nondimensional $Q_{F}, \bar{Q}_{F}=(1+\phi)$, see Eq. 2 et seq.

$Q_{R} \quad$ radiant heat flux

$\bar{Q}_{R} \quad$ nondimensional radiant heat flux

$r \quad$ ratio of reduced Damköhler numbers at extinction with and without radiation, $r=b_{E, R} / b_{E}$. Also, reaction term in Eqs. 2

$\Re$ universal gas constant

$S$ difference of temperature and enthalpy excess, $S=\tau-H$

jí stagnation pnin:

$: \quad$ cime

$T$ temperature

$u$ velocity

$U_{o}(x)$ heaviside step function $U_{0}(x)$ $=\left\{\begin{array}{ll}0, & x<0 \\ 1, & x>0\end{array}\right\}$ 
creation/destruction terms: phenomenology must theretore be abandoned and a set of hinetic equations for soot formation derived. along with a reasonable nucleation criterion.

The approach used herein will be to exam. ine a physically simple model thoroughly. The emphasis will be placed on making mathematically definite statements that can be translated into statements of physical fact, given the limitations of the model. We keep firmly in mind the principle that we are attempting to describe - not simulate - the influence on DFs of radiant heat losses from particulates.

We begin in Sec. II with the formulation of the problem including the heat loss function and the radiation term. In Sec. III we examine the results of the model predictions, including flame movement, extinction formulas, and bounds for radiant extinction. Then in Section IV we discuss the results, indicating points of strength and weakness in the model. The relation of our work to previous studies is discussed in Sec. V. Although literature reviews are usually placed in introductions we felt that for our model the literature review would be more useful if it placed the work in focus after the analysis was complete. Otherwise too many conceptual difficulties should confront the reader at the outset, serving only to obscure the subsequent deductions. We have attempted in most cases to examine the main features of the references we have cited. Finally, Sec. VI presents a short set of point-form conclusions.

\section{FORMULATION}

\section{II.A. Physical Discussion}

The following simplifications are employed in this study: geometrically, we consider the onedimensional "stagnant film" diffusion flame (DF). The porous fuel wall, at temperature $T_{o}$, is iocated at $x-0$, paraliei io the oxicizer waii wibil aiso biz $T-T_{0}$ and is !osicud at $x=i$. The mass fractions of fuel and oxidizer at these walls are $Y_{F F}$ and $Y_{O O}$, respectively (see Fig. 1). Dynamically, we limit ourseives to the case of zero mean flow, so that the movement of species occurs strictly by diffusion. In addition, we neglect the thermophoretic flow that

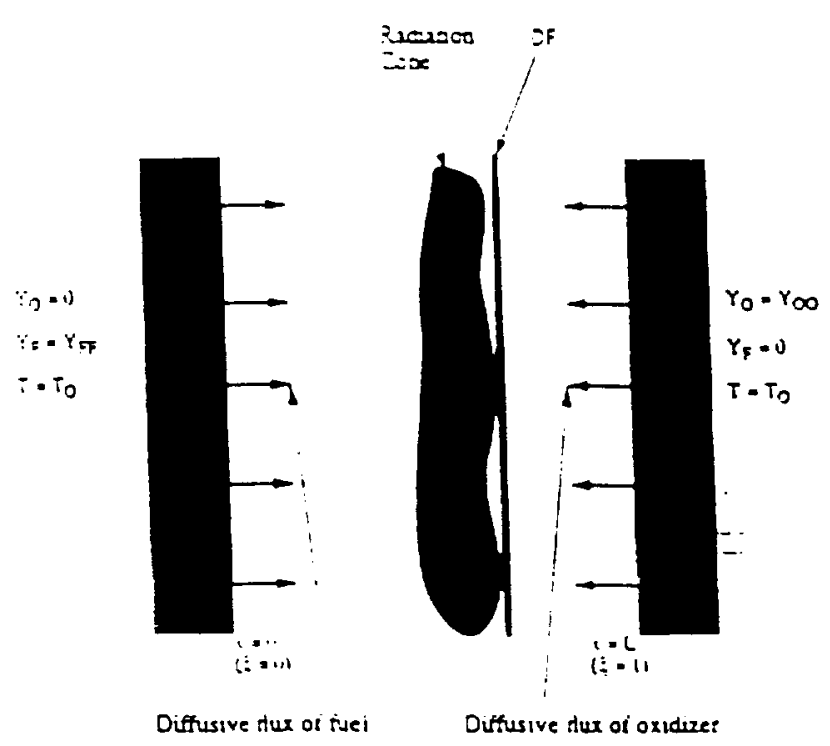

Fig. 1. The physical contriguration for our model problem.

is known to occur with particulates in regions of high thermal gradients, because we wish to examine the thermal and chemical influences of heat losses without factoring in dynamical complications that might make our efforts more difficult. In summary, we have a one-dimensional stagnant-film DF with no mean flow $(u=0)$ and no themophoretic flow $\left(u_{T}=0\right)$. We also impose the steady-state condition $(\partial(\cdot) / \partial t=0)$. For the combustion chemistry we assume that the reaction at the DF occurs through a single irreversible step, $F+\nu O \rightarrow$ $(1+\nu) P$ (on a mass basis), with high activation energy. The "soot-formation mechanism," which we hypothesize occurs on the fuel side of the DF, is assumed not to consume fuel. That is, only "trace" amounts of fuel are required to make "soot" particulates. Also, since the "soot distribution" will be specified, we do not require a separate soot species equation. Nor is it necessary to consider a number-density equation, since we assume that our "soot" particulates are simply a collection of immobile radiating masses located in a preassigned region on the fuel side of the DF. Strictly speaking. iisu is as iecd ever to discuss

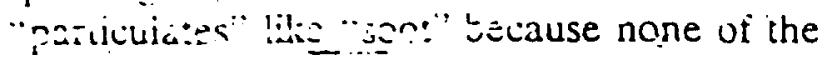
explicit features commonly associated with particulates appear in our analysis. The relevant features of our "soot particulate layer" are exclusively themal; it produces only a region of enthalpy loss that may alter the DF structure and cause extinction. 
b) for the adiabatic Hame temperature. We ulso observe that with heat losses the Hame temperature will assuredly not rise to its theoretical maximum even when $\beta \rightarrow x$. Hence. we may expect a slight redefinition later of the Damköhler number $D$ in terms of a Hame temperature somewhat lower than $T_{f}$.

We note that the derivation of an excess-enthalpy function may be achieved "physically." The conservation equation for the enthalpy takes the form of Eq. 4 when convective transport and body force effects and preferential species transport ( $\mathrm{Le}_{i} \neq 1$ ) are neglected. Since $h=\sum_{i=1}^{N} h_{i} Y_{i}$ and $h_{i}=h_{i}^{\cup}+\bar{C}_{p}\left(T-T^{v}\right)$, it is easy to recover our nondimensional $H$.

Finally, we observe that even in the most difficult and general case, such as when the radiation term depends on the spatial coordinate and the temperature and the fuel mass fraction. as long as no fuel $\rightarrow$ soot depletion terms enter the species equations (Eqs. 1.ii and 1.iii) we can still define the mixture fraction variable $Z=\left(\phi y_{F}+1-y_{O}\right) /(\phi+1)$ that satisfies $Z_{\xi \xi}=0$ with $Z=0$ at $\xi=1$ and $Z=$ 1 at $\xi=0$. This provides an important simplification of the governing equations. The solution for $Z$ is $Z=1-\xi$, whereby

$$
\left.\begin{array}{l}
\text { (i) } y_{O}=(1-Z)-\left(1-Z_{f}\right)(\tau-H), \\
\text { (ii) } y_{f}=Z-Z_{f}(\tau-H)
\end{array}\right\}
$$

where $Z_{f}=(1+\phi)^{-1}$ is the DF. location in the $Z$-coordinate system. Then the equation for $\tau$ (the first of Eqs. 2) and the equation for $H$ (Eq. 4) become

$$
\begin{aligned}
& \text { (i) } \\
& \tau_{Z Z}=-(1+\phi) \operatorname{Dr}(H, \tau, Z) \\
& \div N_{R}\left(-d \bar{Q}_{R} / d Z\right), \quad \tau(0)=\tau(1)=0 \\
& \text { (ii) } \dot{H}_{Z Z} \cdot \ddot{i}_{R}\left(-d \bar{Q}_{R} / d Z\right) \text {. } \\
& H(j)=H(1)=0 \text {. }
\end{aligned}
$$

Hence, the solution for $\tau, y_{F}$, and $y_{O}$ is reduced to the solution of two coupled nonlinear equations, Eqs. 6.1 and 6.ii. Equations 5 and 6 suggest derining $S=:-H$. giving

$$
\text { (i) } \begin{aligned}
& S_{Z Z}=-(1-\dot{0}) D r . \\
& S(0)=S(1)=0 . \\
& \text { (ii) } \quad \begin{array}{l}
H_{Z Z}=V_{R}\left(-d \bar{Q}_{R} / d Z\right), \\
\end{array} \quad H(0)=H(1)=0 .
\end{aligned}
$$

where $r(H, S . Z)=\left[1-Z-\left(1-Z_{i}\right) S\right]$. $[Z-Z f S] \exp [-\beta(1-S-H) /[1-\alpha(1-S$ $-H)]$. It is clear that some rather interesting behaviors may be expected. especially in the general case when the radiation term is a complicated function of $Z, F$, and perhaps other variables. However. we shall examine only the simple case when $\bar{Q}_{R}$ is a prescribed function of $Z$. We shall see that even for this case many complexities arise.

\section{II.C. The Form of $H(Z)$ :}

The enthalpy defect $H(Z)$ is obtained by integrating Eq. 4 or Eq. 7.ii twice. We consider the simple case when the radiant heat transfer term is a known, specified function of position. Then the integrations may be carried out explicitly. Because of the eventual double integration, we do not need to be particular in our choice for the radiant heat flux, $d \bar{Q}_{R}(Z) / d Z$. Hence, we let

$$
-\frac{d \bar{Q}_{R}}{d Z}(Z)=U_{o}\left(Z_{R^{-}}\right)-U_{o}\left(Z_{R^{-}}\right),
$$

as shown in Fig. 2a. The quantities $Z_{R^{-}}$and $Z_{R}$ - are the boundaries of the heat loss zone. We note that $Z_{R^{-}} \geq Z_{f}$ and that there are no restrictions on $Z_{R^{-}}$other than $Z_{R^{*}}<1$, i.e., $Z_{R}$. does not have to be "close" to $Z_{R^{-}}$. From Eq. 8 we see that $d \bar{Q}_{R} / d Z$ is a "well" function, and that $-d \bar{Q}_{R} / d Z$ is a "top-hat" profile. The solution for $H(Z)$ (see Fig. 2b) is

$$
H(Z)=\left\{\begin{array}{l}
0,-0 \leq Z<Z_{R^{-}}, \\
\frac{-\because_{R}}{2}\left(Z \cdot Z_{R^{-}}\right)\left(Z_{R^{+}}-Z\right) . \\
Z_{R^{-}} \leq Z \leq Z_{R^{*}} \\
0, \quad Z_{R^{+}}<Z \leq 1 .
\end{array}\right\}
$$

Note that $H_{Z}=0$ and $H=-N_{R}\left(Z_{R^{*}}-\right.$ $\left.Z_{R^{-}}\right)^{2} / 8$ at $Z=\left(Z_{R^{+}}+Z_{R^{-}}\right) / 2$, also shown in 
This result. used in the definition of $V_{R}$ arter Eqs. 2 and 3. gives

$$
\begin{aligned}
V_{R}= & +\left(\frac{\rho_{0}}{\rho_{R}}\right) L^{*} \kappa_{p}\left(T_{R}, 1\right) \frac{Y_{s R}}{Y_{F F}} \\
& \times \frac{\sigma\left(T_{R}^{+}-T_{0}^{+}\right)}{\rho_{0} \alpha_{o}\left[Q_{F} /(1+\phi)\right] / L^{*}},
\end{aligned}
$$

where $L^{*}=z_{o} / \rho_{o}$ is a length scale of the order of $L, L^{*} / L=L^{-1} \int_{0}^{L}\left(\rho / \rho_{0}\right) d x, T_{f}-T_{0}$ $=Q_{F} Y_{F F} / C_{p}(1+\phi)$ and $\alpha_{0}=\lambda_{0} / \rho_{0} C_{p}$. Hence. $V_{R}$ is the ratio of the blackbody radiant heat flux at $T=T_{R}$ to the flame heat flux, reduced by the length ratio $L^{*} / L_{R A D}=$ $L^{*} \kappa_{p}\left(T_{R}, 1\right)<O(1)$ and the ratio $Y_{s R} / Y_{F F}$.

We shall see in the subsequent developments that it is not $N_{R}$ by itself that is important, but the product of $N_{R}$ and the "soot layer" thickness $\Delta Z_{R}$ in mixture-fraction space.

Numerical estimates for $N_{R}$ can be made. We write $\rho_{\rho} / \rho_{R}=T_{R} / T_{\rho}$ and let $T_{0}=300 \mathrm{~K}$, $T_{R}=2000 \vec{T} \mathrm{~K}$, where $\vec{T}$ is a nondimensional temperature. We also estimate $Y_{S R} / Y_{F F} \sim$ $1 / 20$ and take $Q_{F}=11355 \mathrm{cal} / \mathrm{g}$ (for methane). Then using $\rho_{o}=3 \times 10^{-3} \mathrm{~g} / \mathrm{cm}^{3}$ and $\alpha_{0}=1.24 \mathrm{~cm}^{2} / \mathrm{s}$ we find $N_{R}=$ $1.2 L^{*}\left(L^{*} \kappa_{p}\right)(1+\phi) \bar{T}^{j}$, where $L^{*}$ is in centimeters. We expect $L^{*} \sim 2 \mathrm{~cm}$ and $L^{*} \kappa_{p} \sim$ $O\left(10^{-1}\right)$, whereby $N_{R}=0.14(1+\phi) \bar{T}^{5}$. The factor $1+\phi$ can range from 1 for $\phi<O(1)$ to approximately 20 when $\phi$ is large, so that the quantity multiplying $\bar{T}^{5}$ can range between 0.25 and approximately 5. A $10 \%$ increase of $T_{R}$ (i.e., $\bar{T}=0.9$ ) decreases $N_{R}$ by $40 \%$, giving $N_{R} \sim 0.08(1+\phi)$, whereas a $20 \%$ decrease of $T_{R}$ decreases $N_{R}$ by about $70 \%$. Consequently we expect $N_{R}$ to range from $O\left(10^{-2}\right)$ to $O\left(10^{-i}\right)$ for small $\phi$, to $O(1)-O(10)$ values for large $\phi$.

\section{RESULTS}

\section{Lii. 1 Temperature and Mass-Fraction Frotiles in the Outer (Non-Flame) Regions}

On the oxidizer side $y_{F}=0$ in the lowest approximation, giving $y_{0}=1-Z / Z_{f}$ from Eqs. $5 . \mathrm{i}$ and $5 . \mathrm{ii}$ and $\tau=H+Z / Z$. Now if the "soot" layer is distinct from the reaction zone, i.e.. if $Z_{p^{-}}>Z$. it has no infiuence on the temperature on the oxidizer side and $\tau=Z / Z$. $\Rightarrow d \tau / d Z=1 / Z$; there. In this case the oxjdizer side is exactly the same as for a "nonsooting" flame. If. however. the leftmost edge of the soot laver and the Hame zone slightly

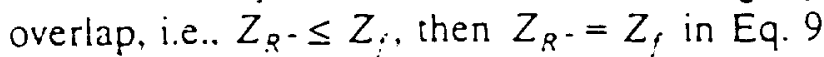
(we anticipate that due to "oxidation" the difference between $Z_{R^{-}}$and $Z_{i}$ will be small), giving

$$
\tau=\frac{Z}{Z_{f}} \div\left\{\begin{array}{l}
0 . \quad 0 \leq Z<Z_{R^{-}}=Z_{f}, \\
-\frac{N_{R}}{2}\left(Z-Z_{f}\right)\left(Z_{R^{-}}-Z\right), \\
Z_{R^{-}} \leq Z .
\end{array}\right\}
$$

and

$\frac{d \tau}{d Z}=\frac{1}{Z_{f}}+\frac{d H}{d Z}=\frac{1}{Z_{f}}-\frac{N_{R} \Delta Z_{R}}{2}$

as $Z$ approaches $Z$ from the oxidizer side. Hence, the influence of the heat losses is to decrease the temperature gradient on the oxidizer side from its undisturbed value, $1 / Z_{f}$. We observe that since $d \tau / d Z$ must be positive we obtain an upper bound for $\left.N_{R}\right\lrcorner Z_{R}, N_{R} \Delta Z_{R}$ $\leq 2 / Z_{f}=2(1+\phi)$. This criterion may be interpreted as stating that when the heat losses become large the thickness $\Delta Z_{R}$ can decrease: in other words, as $N_{R}$ increases we no longer need a thick "soot" layer to produce large heat losses from the flame. This argument can, of course, be generalized to parameters within $N_{R}$. For instance, suppose that $N_{R} \Delta Z_{R}$ at extinction has been determined and that $\Delta Z_{R}$ is fixed, thereby fixing $N_{R}$. Consequently if $T_{R}$ is changed at constant pressure, constant stoichiometric index $\phi$, constant $Q_{R}$, constant $b$, and so on, we must have $\kappa_{p}\left(T_{R}, 1\right) Y_{s R} T_{R}{ }^{5} \approx$ constant; if $\kappa_{p}=$ constant, then a slight increase (or decrease) of $T_{R}$ can be accompanied $h \because n$ large decrease (or increase) of $Y_{j}$. Physically. as the mean "sout" icyer cemptiulure increases fewer soot particles are needed to produce the same heat loss. $A 10 \%$ increase of $T_{R}$, for example, decreases $Y_{s R}$ by $38 \%$.

We now examine the fuel side. Here we have $y_{O}=0$ in the lowest approximation, giving $y_{F}=\left(Z-Z_{f}\right) /\left(1-Z_{f}\right)$ and $\tau=H+1$ 
coward the tuel side. We demonstrate this by writing the Hame location as

$$
Z_{i}=\frac{1}{1-\phi}=1-\frac{z_{1}}{z_{0}}=1-\frac{i_{1} \rho d x}{i_{1}^{L} \rho d x} \text {. }
$$

This can be rearranged to yield

$$
\int_{1)}^{x} \rho d x=\phi \int_{x_{f}}^{L} \rho d x
$$

which we examine for the cases with and without heat losses. If the tlame zone is negligibly thin we can write $\rho=\rho_{o}-\left(\rho_{o}-\rho_{f}\right)(L-$ $x) /\left(L-x_{f}\right)$ on the oxidizer side. giving $\rho\left(x_{f}\right)$ $=\rho_{f}$ and $\rho(L)=\rho_{o}$ and vielding $\phi(L-$ $\left.x_{j}\right)\left(\rho_{o}+\rho_{f}\right) / 2$ for the RHS of the above equation. For the LHS (the fuel side) we can use two different $\rho$ distributions. the linear profile without heat losses.. $\rho^{(1)}=\rho_{o}-\left(\rho_{o}-\right.$ $\left.\rho_{f}\right)\left(x / x_{f}\right)$, and a nonlinear profile with losses, $\rho^{(2)}=\rho_{o}-\left(\rho_{o}-\rho_{f}\right)\left(x / x_{f}\right)^{2}: \rho^{(2)}$ is always larger than $\rho^{(1)}$. Substitution into the LHS above yields $x_{f}\left(\rho_{0}+\rho_{f}\right) / 2$ and $x_{f}\left(\rho_{0}+2\left(\rho_{0}\right.\right.$ $\left.\left.+\rho_{f}\right) / 2\right) / 3$, respectively, from which we find, after equating to the RHS, $\left(x_{f} / L\right)^{(1)}=\phi /(1$ $+\phi)$ and $\left(x_{f} / L\right)^{(2)}=\bar{\rho} \phi /\left(\rho_{o} / 3+\bar{\rho}(\phi+\right.$ $2 / 3)$ ), where $\bar{\rho}=\left(\rho_{o}+\rho_{f}\right) / 2$. We then form the difference.

$$
\begin{aligned}
& \left(\frac{x_{F}}{L}\right)^{(1)}-\left(\frac{x_{f}}{L}\right)^{(2)}=\frac{\phi M}{(1+\phi)(1+\phi+M)} \\
& M=\frac{1}{3}\left(\frac{\rho_{o}-\rho_{f}}{\rho_{o}+\rho_{f}}\right)>0 .
\end{aligned}
$$

Hence, case 2 with heat losses has a smaller value of $x_{f} / L$, indicating that the flame is displaced to the fuel side. Although our demonstration has employed simple $\rho$ distributions, it is generally valid whenever $\rho$ on the oxidizer side is unchanged by the heat losses and when the nondimensional reaction layer thickness, $\Delta x_{f} / L$, is small.

Becausc uur analysis is pertormed in terms

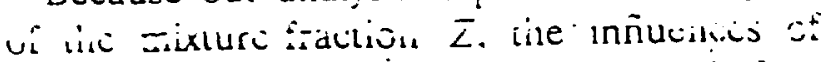
heat losses on $Z_{f}$ must be examined before making definite conclusions. In any case, the flame movement caused by density changes does not alter the flame location in the $Z$ coordinate, since $Z_{f}=(1+\phi)^{-1}$ is unchanged.

\section{III.C. The Chemical Reaction Zone}

First we analyze the case $Z_{R^{-}}=Z_{f}$, so that $H\left(Z_{.}\right)=0$. We employ Eqs. 7.i. 9 with $Z_{R^{-}}=$ $Z, 11$. and 12 . The use of the "S-equation" is of considerable benerit. because in all results except the presence of $H$ in the exponential it resembles the standard DF "structure" equation [1]. The gradients of $S$ in the outer zones are identical to those for the zero heat-loss case, viz $S_{Z}=(\tau-H)_{Z}=1 / Z_{f}$ on the fuel side (compare to Eq. 11 for $\tau_{Z}$ ) and $S_{Z}=(\tau-$ $H)_{Z}=-1 /\left(1-Z_{f}\right)$ on the oxidizer side (compare to Eq. 12). We therefore define stretched variables,

$$
\left.\begin{array}{l}
\text { (i) } S=1-(\Phi+a \eta) / b \beta, \\
\text { (ii) } \quad \eta=\beta\left(Z-Z_{f}\right) c,
\end{array}\right\}
$$

as for standard DF asymptotic analysis [2]. We observe that $S$ is presumed to approach unity in the reaction layer since $S=\tau-H$ and $H$ $=0$ there, enabling $\mathrm{T} \rightarrow 1$ as $\beta \rightarrow \infty$. We shall subsequently see that when $H\left(Z_{f}\right) \neq 0$ we may still use Eq. 13.i because this implies $\tau_{\max }=1$ $+H\left(Z_{f}\right)<1$, so that the flame temperature cannot attain its zero-heat-loss peak value. With the above substitutions the quantity in the argument of the exponential of Eq. 7.i becomes

$$
\begin{aligned}
& -\frac{\beta(1-S-H)}{1-\alpha(1-S-H)} \\
& \quad \frac{[-(\Phi+a \eta) / b+\beta H]}{\left[1-\frac{\alpha}{\beta}\left\{\frac{\Phi+a \eta}{b}-\beta H\right\}\right]}
\end{aligned}
$$

which is in danger of being swamped by the heat loss term $\beta H$ if appropriate measures are not taken. For $Z$ near $Z_{f}$, we write

$$
\begin{aligned}
H(Z)= & H\left(Z_{i}\right)-\left(Z-Z_{i} i \frac{\sin \left(\bar{L}_{i} j\right.}{d L}\right. \\
& +\frac{\left(Z-Z_{f}\right)^{2}}{2 !} \frac{d^{2} H\left(Z_{f}\right)}{d Z^{2}}+\ldots
\end{aligned}
$$

With $H\left(Z_{f}\right)=0$, and $d H\left(Z_{f}\right) / d Z=$ $N_{R} \Delta Z_{F} / 2$, and Eq. 13.i., we find $H(Z) \doteq$ 
When $H\left(Z_{0}\right) \neq 0$. there is real overlap beween the flame and the "soot" laver. so that $Z_{Q}<Z_{\text {. }}$. Vevertheless. the preceding analysis carries through in almost the same manner if we replace $\beta$ in Eqs. 13 .i and 13 .ii by $\beta_{R}=$ $\beta T_{i}, T_{i R}$. Here $T_{j R}$, the flame temperature with radiant heat losses. is smaller than $T_{i}$. It is obtained from the relation $\tau_{R}=1 \div H\left(Z_{i}\right)$, giving $T_{f R} / T_{f}=1+\alpha H\left(Z_{f}\right)$. The Arrherius exponent becomes

$$
\begin{aligned}
& -\frac{\beta(1-S-H)}{1-\alpha(1-S-H)} \\
& \quad=-\frac{\beta\left(1-\tau_{R}\right)}{1-\alpha\left(1-\tau_{R}\right)}-\frac{1}{b}\left[\Phi+a_{R} \eta\right] \\
& \quad+O\left(\beta^{-1}\right) .
\end{aligned}
$$

The term $-\beta\left(1-\tau_{R}\right) /\left[1-\alpha\left(1-\tau_{R}\right)\right]=$ $-(E / H)\left(1 / T_{f R}-1 / T_{f}\right)$ produces a modified Damköhler number, $D_{R}=D \exp [-(E / \mathcal{H})$. $\left.\left(1 / T_{f R}-1 / T_{f}\right)\right]$, that is smaller than $D$. After some algebra we once again obtain the problem given by Eqs. 14, with $a_{R}$ given by Eq. 15 . $a=2 Z_{f}-1, b / c=2 Z_{f}\left(1-Z_{f}\right)$, and $b=$ $\left[4(1-\phi) D_{R}\left(Z_{f}\left(1-Z_{f}\right) / \beta_{R}\right)^{3}\right]^{1 / 3}$, in which $D$ and $\beta$ have been replaced by $D_{R}$ and $\beta_{R}$. We note that since $D_{R}<D$ and $\beta_{R}>\beta$ the present reduced Damköhler number is always smaller than for the non-"sooting" flame.

\section{III.D. Extinction Criteria}

We deduce the criteria for the DF. with heat losses from the "soot" layer. There are at least two ways to do this. Both produce the same extinction zone on an $N_{R} \Delta Z_{R}$ vs. a (or $Z_{f}$ ) plot. but the second method allows more physical interpretation.

In the first method we observe from Eq. 16 that (i) $-1<a_{R}<+1$ and (ii) $0 \leq\left|a_{R}\right|<1$. Since $\left.a_{R}=2 Z_{f}-1+\left(N_{R}\right\lrcorner ?_{R}\right) Z_{f}\left(1-Z_{f}\right)$. We hiala tos criterion (i) after a shor algebraic caiculation that $1 / 2<Z_{f}<1$ gives $0<$ $N_{R} \sqcup Z_{R}<1 / Z_{f}$ and $0<Z_{f}<1$ gives $0<$ $N_{R} \Delta Z_{R}<2 / Z_{F}$. Thus, for the entire range of $Z_{f}, N_{R} \Delta Z_{R}$ must be positive and smaller than $2 / Z_{f}$. With criterion (ii) we are able to produce a lower limit for $N_{R} \Delta Z_{R}$ that exceeds zero when $0<Z_{f}<1 / 2$. We find the same limits for $﹎{R}\lrcorner Z_{R}$ as in (i) when $1 / 2<Z:<1$ but when 1$)<Z,<1=$ We obtain 2 (1 2Z.) $\left./\left(Z .\left(1-Z_{1}\right)\right)<v_{R}\right\lrcorner Z_{F}<2 / Z$. The extinction boundaries deduced here are shown in Fig. t. They bound the lined region. The width of the extinction interial varies from 2 as $Z_{i} \rightarrow 0$ to + at $Z_{j}=1 / 2$ back to 2 as $Z_{f} \rightarrow 1$. Observe also that extinction appears to be easier when $1 / 2<Z_{1}<1$ because the $N_{R} \Delta Z_{R}$ values required are fairly small. The physical reason for this is that the heat losses by conduction from the reaction zone to the fuel side are greater than to the oxidizer side when $1 / 2<Z_{f}<1$. We see this by writing Eq. 16 as $1-a=2 /(1+\gamma)$, where $\gamma=\left|\tau_{Z}\right|_{\text {fuel }} /\left|\tau_{Z}\right|_{\text {ox }}$; hence, $\gamma>1$ when $0<a<1$. When the "soot" layer is on the fuel side the heat losses to it are amplified. making extinction easier. When $0<$ $Z_{f}<1 / 2$ these two lactors act in opposition. making extinction more difficult.

In the second method we examine the extinction criteria for Eq. 14 in detail. It is well known that for a fixed value of $a_{R}$, Eq. 14 behaves as follows: when $b>b_{\varepsilon}$, where $b_{E}$ is the "extinction" Damköhler number, there are two solutions, only one of which we can accept as "physically realistic". When $b<b_{E}$ there are no solutions. Each value of a produces a single value of $b$, enabling the determination

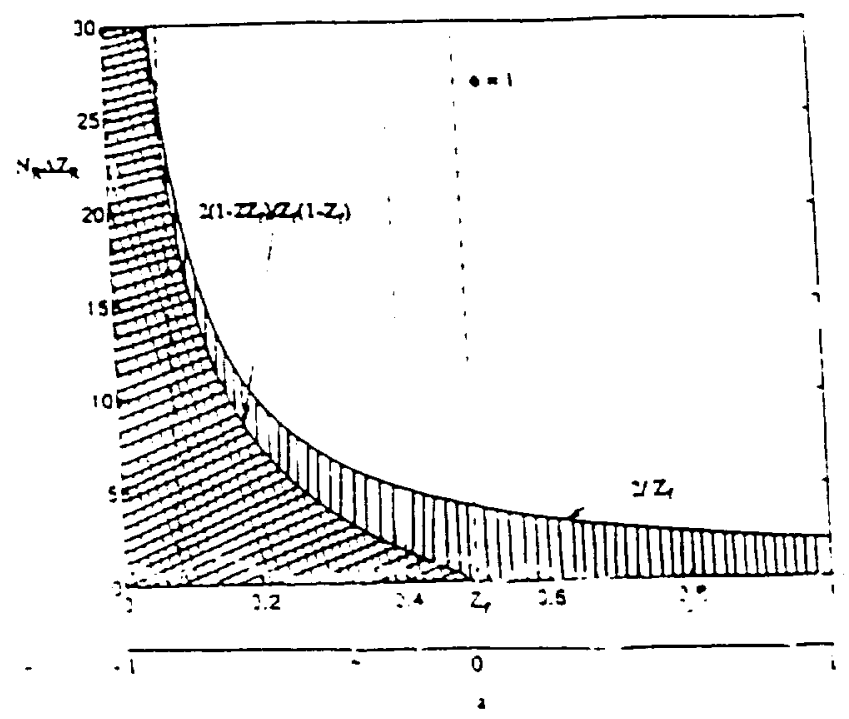

Fig. 4. $N_{R} \perp Z_{R}$ versus $a$ and $Z_{f}$. When $T_{f R . E}<T_{f . E}$ extinction can occur only in the lined region. The dastied vertical lines at $Z_{f} \sim 0.08$ and $Z_{f} \sim 0.4$ indicate bounds proposed by Kennedy et al. [14], outside of which they assert that the soot growth rate is zero. When $T_{f R . E}>T_{f, E}$ extinction must occur in the cross-hatched region. 
At extinction we can equate our two racios $r \equiv 10$ obtain

$$
\begin{aligned}
r_{E} & =\frac{f\left(1-a_{R}\right)}{f(1-a \|)} \\
& =\frac{T_{f R . E}}{T_{f . E}} \exp \left(\frac{-E}{3) H T_{f . E}}\left(\frac{T_{i . E}}{T_{f R . E}}-1\right)\right) .
\end{aligned}
$$

There are two cases, $T_{f R . \varepsilon}<T_{f, \varepsilon}$ and $T_{f R . E}>$ $T$ T.

$$
\text { Case (i) } T_{f R . E}<T_{f, E}
$$

Here we have $r_{E}<1$. This can be satisfied only when $1-\left|a_{R}\right|<1-|a|$, or $\left|a_{R}\right|>|a|$ (see Fig. 5). We recall from Eq. 15 that $a_{R}>a$ is always rue. Hence, extinction can occur only when the two inequalities $a_{R}>a$ and $\left|a_{R}\right|>|a|$ are satisfied. When $a \geq 0$ these inequalities are obviously satisfied and produce the admissible range of values $\left.0 \leq N_{R}\right\lrcorner Z_{R} \leq 2 / Z_{f}=4 /(1+$ a). When $a<0$ (or $0<Z_{f}<1 / 2$ ) we always have $a_{R}>a$, but $\left|a_{R}\right|>|a|$ requires $\left|a_{R}\right|=a+$ $\left(N_{R} \Delta Z_{R}\right) Z_{f}\left(1-Z_{f}\right)>|a|=-a$, whereby $\left.N_{R}\right\lrcorner Z_{R}>8(-a) /\left(1-a^{2}\right)=2\left(1-2 Z_{f}\right) /$ $\left(Z_{f}\left(1-Z_{f}\right)\right)$, which is the same set of limits deduced before. In summary,

$$
\left.N_{R}\right\lrcorner Z_{R}>\left\{\begin{array}{c}
\frac{Z\left(1-2 Z_{f}\right)}{Z_{f}\left(1-Z_{j}\right)}=\frac{8(-a)}{1-a^{2}} \\
0<Z_{j} \leq \frac{1}{2}, \\
0 ; \\
\frac{1}{2} \leq Z_{f}<1,
\end{array}\right\}
$$

and

$$
\begin{aligned}
& N_{R} \unlhd Z_{R}<\frac{2}{Z_{f}}=\frac{4}{1+a} \\
& 0 \therefore \dot{L}_{i}<1 \quad(-1-a<1) .
\end{aligned}
$$

This produces the same lined region of Fig. 4 as previously, but with the added restriction $T_{f R . E}<T_{f, E}$. Here the DF with heat losses extinguishes at a lower flame temperature than the DF without heat losses.
Case (ii): $T_{j R . E}>T_{\therefore \varepsilon}$

Here we have $r_{-}>1$. so that $a_{R}>1$ and $a_{a_{3}}$ $<|a|$. These conditions restrict us to $-1<$ a $<0(0<Z$. $<1 / 2)$. Where we tind the lower limit $\left.v_{R}\right\lrcorner Z_{R}=0$ and the upper limit.$\left._{R}\right\lrcorner Z_{Q}$ $=2(1-2 Z),.[Z .(1-Z)$.$] , which cuincides$ with the lower limit in Eq. 23 for the case $-1<a<0$. This extinction region is shown in Fig. 4 as the cross-hatched zone.

We therefore have demonstrated that this approach allows us to describe the region below $N_{R} \perp Z_{R}=2 / Z_{f}$.

Our results raise two questions. (1) Does the region produced by $T_{f R . E}>T_{f . \varepsilon}$ make "physical sense"? This is debatable. because for conditions other than extinction Eq. 20 demonstrates that $r\left(Z_{j}\right)=b_{R} / b$ is always less than unity. How then is it possible for $b_{R} / b$ at extinction to suddenly rise to above-unity values? We suspect that this region is indeed nonphysical. (2) Can fuel-poor DFs with $\phi<1$ (or $1 / 2<Z_{f}<1$, or $0<a<1$ ) even produce enough "soot" to self-extinguish? According to our model they can indeed. In practice. however, the condition $\phi<1$ places tight restrictions on possible extinction conditions. For the general hydrocarbon-oxygen reaction

$$
\begin{gathered}
C_{n} H_{m}+(n+m / 4)\left(\mathrm{O}_{2}+x \mathrm{~N}_{2}\right) \\
\rightarrow n \mathrm{CO}_{2}+(m / 2) \mathrm{H}_{2} \mathrm{O} \\
+(n+m / 4) x \mathrm{~N}_{2},
\end{gathered}
$$

we have $v=32(n+m / 4) /(12 n+m)$. which ranges from 3.08 to 4.23 when $m / n$ ranges from 1 to 5 . Hence, $\phi<1$ implies $Y_{F F} / Y_{O O}<$ $1 / \nu$, which ranges from $1 / 4$ to $1 / 3$. For air $\left(Y_{O O}=0.23\right)$ this means $Y_{F F} \in(0.055 .0 .075)$. which is a rather narrow interval, possibly below the extinction limit and certainly so fuel poor that expectations of "soot" Froduction may be in vain. For elevated-oxigen environments peitaps elivugn "suor" may form to weaken the DF.

On the fuel-rich side of Fig. 5 the "physically realistic" interval for extinction (the lined region in Fig. 4) becomes progressively more difficult to attain as $Z_{f}$ decreases. For $\mathrm{HC}$-air 


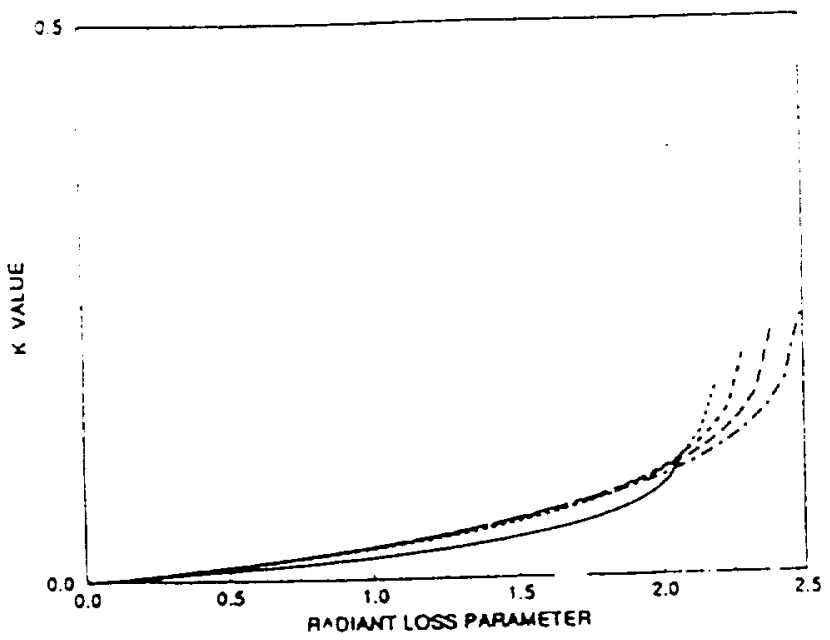

Fig. 6. (c) Same as (a) except $Y_{F F}=1$.0. The lines are heavier than in (b).

0.2 to 1.0 in increments of 0.2 . Thus, $Z_{f}$ ranges from 0.2 to 0.55 . The abscissa is $N_{R} \Delta Z_{R}$ $\left(N_{R} \backslash Z_{R}\right)_{\min }$, which has a range of 2.5 when $Z_{f}=0.2$, a range of 4.0 when $Z_{f}=0.5$ and a range of only 3.6 when $Z_{f}=0.55$; this is why the trend to increased range of the successive curves for $Y_{O O}=0.2,0.4,0.6$, and 0.8 is broken when $Y_{O O}=1.0$. In Fig. $6 \mathrm{~b}$ we have $Y_{F F}=0.6$ with $Y_{O O}=(0.2,0.4,0.6,0.8,1.0)$. Here the range of $N_{R} \Delta Z_{R}$ continually increases. The same behavior is found in Fig. 6c, where $Y_{F F}=$ 1.0. The data of Figs. $6 a-6 c$ are normalized by dividing by the maximum value of each final data point for each curve. This normalization improves the correlation of the data as $Y_{F F}$ increases, see Fig. 6d. More sophisticated normalizations are clearly possible, but this is an exercise we do not undertake here.

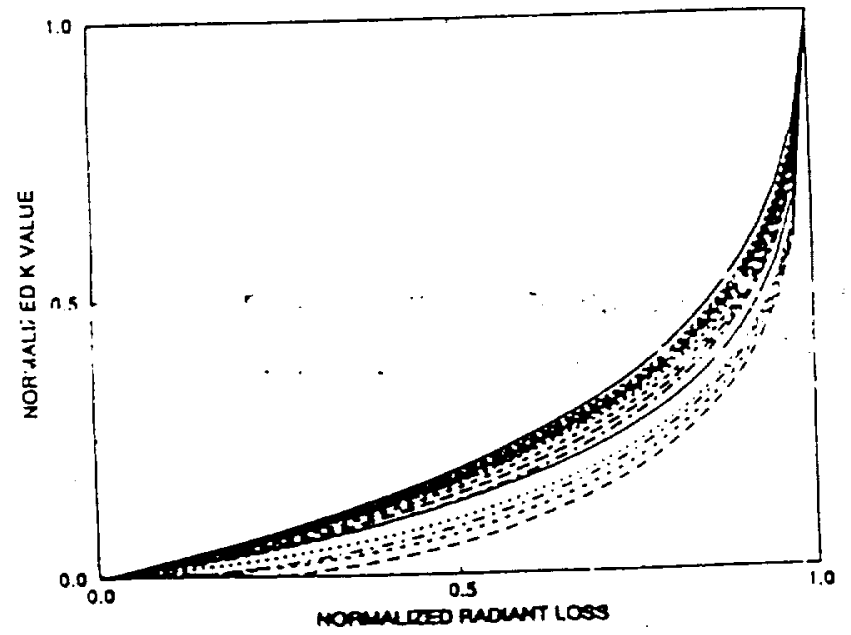

Fig. 6. (d) Normalized data of (a), (b), and (c).
TABLE I

Parameter Values L'sed to Generate Figs. 6 and 7

\begin{tabular}{cl}
\hline Parameter & Vumerical Value \\
\hline$E$ & $29.100 \mathrm{cal} / \mathrm{mol}$ \\
$C_{F}$ & $19.323 \mathrm{cal} / \mathrm{g} \cdot \mathrm{K}$ \\
$Q_{F}$ & $11.355 \mathrm{cal} / \mathrm{g}$ (fuel) \\
$\nu$ & $: .0$ \\
$T_{0}$ & $239 \mathrm{~K}$ \\
\hline
\end{tabular}

It is perhaps more useful to focus on $\Delta=$ $\alpha\left|H\left(Z_{f}\right)\right|$ since this is the temperature decrement produced by the heat-loss zone. For the same $Y_{F F}$ and parameter values, the results for $\Delta$ vs. $N_{R} \Delta Z_{R}-\left(N_{R} \Delta Z_{R}\right)_{\min }$ are shown in Figs. $7 a-7 c$. Observe the similarity to Figs. 6a-6c. The correlation analogous to Fig. $6 \mathrm{~d}$ is shown in Fig. 7d.

\section{DISCUSSION OF RESULTS}

We have made numerous simplifications during our analysis. It is necessary to examine these in order that future studies might introduce the necessary refinements.

The radiation term in Eq. 1 (or Eq. 4, or Eq. 7.ii) was simplified by ignoring its functional dependence on $\tau, y_{F}$, etc. It was replaced by the simple model function of Fig. $2 \mathrm{~b}$ that allows decoupling of Eq. $7 . i$ and $7 . i i$ and facilitated the subsequent analysis. Later, in Sec. II.D, we assumed the soot layer was an optically thin medium, which allowed us to relate $d \bar{Q}_{R} / d Z$ in Eq. 7.ii to the soot mass fraction $Y_{S}$

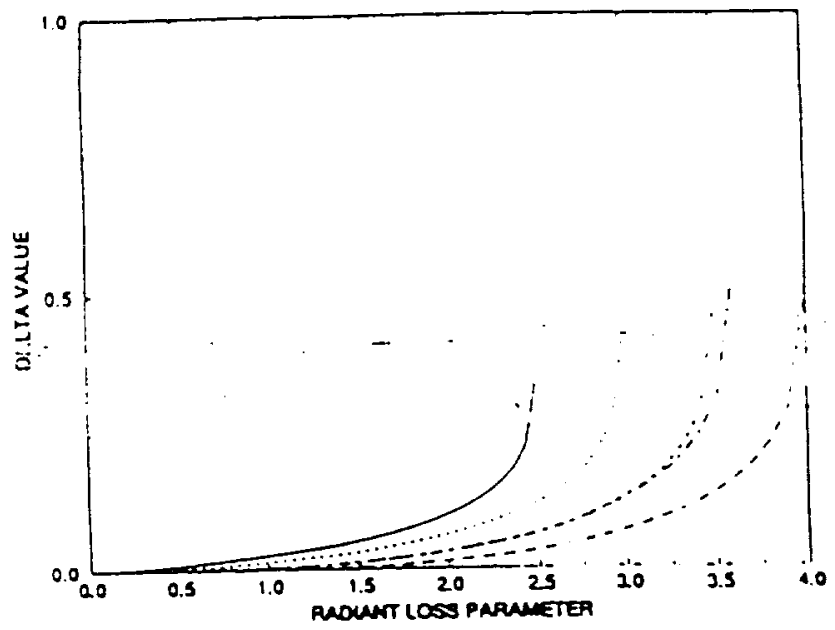

Fig. 7. (a) $\Delta=\alpha\left|H\left(Z_{f}\right)\right|$ versus $N_{R} \Delta Z_{R}-\left(N_{R} \Delta Z_{R}\right)_{\text {rias }}$ Same conditions as Fig. $6 \mathrm{a}$. 
limit when $x_{y^{-i}} d(=) / d x \ll 1$. The advantage of this formulation is that the integrodifferential equation of radiant transter is replaced by a nominally tractable ordinary differential equation. This might enable analytical solutions to be found. or. at least. numerical results to be obtained without employing sophisticated and expensive computational software and hardware. Just as importantly, it preserves the modeling imperative, the notion that in modeling we wish to describe what happens. not simulate it.

From these discussions of the purely radiative features we see that much "structure" for $S$ and $H$ remains to be uncovered from Eqs. $7 . i$ and $7 . i i$. We have, through the specification of $H(Z)$, produced a simplified theory that only touches the surface of this "structure."

The approximation made for the reaction chemistry was standard, in contrast with studies that include detailed chemistry. In order to produce a model for the chemical reaction processes that describes fuel breakdown and soot formation, however, we do not require a detailed numerical simulation, unless we are certain that soot production is initiated upon the appearance of a specific "precursor." All we require is a qualitative reaction sequence that resembles the actual sequence in its most important features. With our fast-chemistry model, we determined that the "soot" layer had to touch the reaction zone to influence it. This conclusion may change when more than trace amounts of fuel are required to produce "soot", but the implications are clear: the dependence in the reaction zone on $T$ through the Arrhenius factor $\exp (-E / \Re T)$ is strong enough to drive the chemical reaction to completion even when broad regions of heat loss exist nearby. Only when the losses severely intermpt the zone of maximum reaction rate, which for $\phi<1$ (fuel-poor or oxygen-rich) occurs slightly to the oxidizer side of $Z_{f}$, and for $\dot{\omega}>1$-occurs slightly to the iuel side of $Z_{f}$,

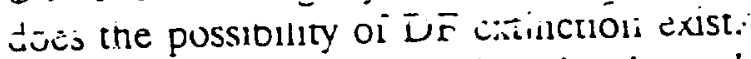

The extinction mechanism is thermal because the sole influence of "soot" is to create a heat-loss zone. In fact, there is no reason to presume that our virtual radiating particulates have their origins in soot. The heat-loss zone is generic and may perhaps be best thought of as follows: imagine a collection of small chemically inert spherules distributed on the fuel side of the domain. because oxidation destroys them when they penetrate the Hame. Above a certain temperature they radiate energy out of the domain $0<x<L$. We may assume that the heat flux leaving each point of the heat-loss zone is identical. giving for $-d Q_{R} / d Z$ the "top-hat" profile already discussed in Sec. II.D. This amounts to defining a characteristic losszone temperature $T=T_{R}$, viz, $T_{R}\left(T_{R}{ }^{4}-T_{0}{ }^{4}\right)$ $=\Delta Z_{R}{ }^{-1} i_{Z_{R}-}^{Z_{Z^{-}}} T\left(T^{4}-T_{0}^{4}\right) d Z$; the constancy of $Y_{S}$ on the ruel side would eliminate the need for a "soot" mass protile like the one described by the "top hat" profile of Fig. 2a. The result would be a radiant heat-loss zone between two temperatures (the minimum, $T_{S \text { min }}$ and the maximum. $T_{f R}$ ) separated by the distance $\Delta Z_{R}$ in mixture fraction space.

In this article we have moved the "soot layer" at will or, reverting to the above intepretation, we have defined the bounding temperatures of the heat-loss layer at will. Heat-loss regions in real DFs, however, usually locate themselves through a web of interactions involving nucleation. particle growth, convective flow, thermophozetic flow, etc. If we presently ignore the details of nucleation and particle growth and assume that mature particles spontaneously appear. we must still account for the imbalance of physical forces. In our siudy we have neglected convection and thermophoresis. It is known that convective flows of fuel toward the DF and thermophoretically induced particulate motion away from the DF strike a balance that dictates the soot layer location. Oddly enough, without convection but with thermophoresis, the stable condition for our problem is a steady thermophoretic flow of particulates toward the fuel wall. Thus, the entire fuel side would be populated with carbon particulates streaming steadily towards the fiel wall. This suggests locating the soot zone according to strictly the mad Giveriz. which in fact appeurs ic ie the noji pibyusal!y seif-consistent way to interpret the model examined in this article.

For the flow field there are many complications. In this study they have been eliminated by ignoring the flow, though it would be relatively straightforward to introduce a convective 
portant: increase it to form more soot. decrease it to produce less soot.

Kent et al. [9], with their Wolfhard-Parker burner. tound that the soot particulates are generated very close to the reaction zone. that the soot formation rate has its peak values about $2-3 \mathrm{~mm}$ from the reaction zone on the fuel side, that the soot volume fraction and particle diameter profiles peak at about $5 \mathrm{~mm}$ on the fuel side of the $T$-peak, that near the flame base the soot formation rate becomes negative (oxidation), and that the soot particulates can be convected through the DF.

The 1985 study by Smyth et al. [10] has an extensive bibliography ( 59 references) and certain succinct and direct conclusions, one of which we now quote. With their WolfhardParker bumer for $\mathrm{CH}_{4}$ /air DFs these authors determine that ". . condensation reactions are found in a localized region approximately 2-3 $\mathrm{mm}$ on the fuel side from the zone of maximum temperature. These intermediate $\mathrm{HCs}$ exhibit maximum concentrations at temperatures of approximately $1300-1650 \mathrm{~K}$. The earliest soot particles are detected at the high temperature edge of this region, which is rich in aromatic compounds and other unsaturated HCs." The Kent et al. [9] and Smyth et al. [10] studies are in general agreement over the proximity of the soot layer to the reaction zone. But Wolfhard-Parker bumers have substantial rates for free and forced convection, perhaps negating the thermophoretic flows and producing a bias towards proximate soot layers and DFs.

The studies of Santoro and Colleagues examined soot growth along individual particle paths. They used a coannular DF and performed extensive particle, temperature and velocity-field measurements using laser velocimetry (LV) [11]. They estimate a maximum thermophoretic velocity $u_{T \max }$ of $4 \mathrm{~cm} / \mathrm{s}$ and observe that in the region where $u_{T}=u_{T \max }$ iic radial veics:- angas herrecil iu and so

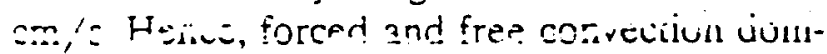
inate thermophoresis. Soot is formed in the annular region of the flame, where the soot volume fractions are maximum, $\Phi_{\max }$. As the burner flow rate increases the path line for $\Phi$ "shifts" outward to larger radial values. The velocity field is dominated by buoyancy, mak- ing it insensitive to changes in the fuel flow rate. but the soot field is strongly dependent on 1. through the fuel flow rate. The nwo major contributions to increased soot formation are increased ?, and changes in the flame shape caused by the "shift" of $\Phi_{\text {max }}$. Increases in the fuel how rate do not increase soot formation rates. The values of $\Phi_{\max }$ are $O(10)$ to $O(100)$ times larger than near the centerline of the flame axis. except for the region close to the flame tip, suggesting that soot is indeed able to penetrate (diffuse?) to the center of the flame cone given sufficient time. Unfortunately, there is no information on the figures of the relative locations of the soot, flame, and oxidation regions. Nevertheless, it is clear that increased fuel flow rates enable soot particles to move on path lines with larger radial values, indicating that regions of soot formation respond to a change in flow rates.

A recent study that supports this statement is that of Jackson et al. [12], for $\mu g$ droplet burning of heptane and heptane/monochloroalkane mixtures. The latter fuel soots more than the former because the $\mathrm{Cl}^{+}$radical "scavenges" the $\mathrm{OH}^{-}$atoms that usually inhibit soot formation. These authors suggest that the spherical "soot shell" is relatively close to the droplet surface. Hence, contrary to the previous studies [8-11] it appears the inwardly directed thermophoretic forces can compete with the outward convective flow. They indicate that "soot shell" formation occurs where $F_{D}+F_{T}=0$, where particle drag and thermophretic forces cancel, that the force balance depends on agglomerate size and gas properties (mostly the viscosity $\mu$ and the diffusion coefficient $D$ ), and that the soot formation can be minimized by ensuring the formation of smaller particulates that do not break through the DF, but are consumed by it. This study raises questions about the thermophoresisconvection competition. The forre balance suggests iilai inermophoresis can be very impor-

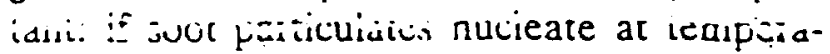
tures above $1650 \mathrm{~K}$ (as suggested in (10), there must be a rather vigorous migration from the high- $T$ near-DF region to the low- $T$ nearsurface region. Can such rapid migration away from the regions abundant in aromatic compounds and unsaturated HCs [10] still allow 
not include $T$ in their calculation of $\Phi$. so that although $T$ drops once the Hame is crossed from the fuel to the oxidizer side. the corresponding drop in $\phi$ is much smaller than it might be. An ad hoc attempt to include the $T$ effect (which they say simulates the temperature drop due to radiation) improves the agreement only slightly. They also find that $\Phi$ depends strongly on $k$. increasing by a factor of 10 when $k$ increases only by a factor of 2.5 . They also note that $k$ is actually a function of $Z$ and time $t$, and that $k$ decreases as $t$ increases. because "young" soot has a higher surface growth rate than "old" soot. They conclude by drawing attention to the need for accounting more precisely for the influences of radiation on the $T$ distribution in sooting flames. A means must be found to introduce variable $T$ into the equations for $\Phi, N$, etc.. and radiative loss terms as functions of $\Phi . N, \ldots$ must also be derived.

The study of Pagni and Okoh [15] examines certain fundamental questions of soot-flame interaction using a two-dimensional laminar boundary-layer flame on a pyrolyzing slab. This is really a multidimensional version of Fig. 1 . Perhaps because of the strong convective flows they find negligible thermophoretic influences. They employ a simplified phenomenological expression of Arrhenius form to describe the soot growth rate. Based on existing empirical data they state that the .... optically thin approximation is valid for incorporating particle radiation in the gas boundary layer equations."

The previous study with perhaps the most relevance to our work is that of Eeung et al. [16]. It advances a mechanism for soot formation in DFs that with refinement should be able to eventually absorb the phenomenological models. The authors begin by making several observations: (i) "...measurements indicate that soot formation is dependent on the breakdown path of the fuel and the presence or pyrolysis products sucn as aceivlente. aint

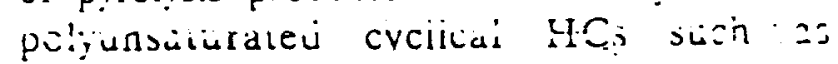
benzene..." (ii) pyrolysis products "iend to show similar profiles with different magnitudes...," so that numerous "critical species" exist, like $\mathrm{C}_{2} \mathrm{H}_{2}, \mathrm{C}_{6} \mathrm{H}_{6}, \mathrm{C}_{4} \mathrm{H}_{6}$, etc.; and (iii) the detailed models are too complex to use in "flows of practical importance for years to come" and the global (phenomenological) models are of dubious validity "under conditions different irom those under wich they were originally tormulated." They construct a soot formation mechanism for DFs containing fice components. The first component is the chemical reaction mechanism that predicts at least qualitatively correct levels of $\mathrm{C}_{2} \mathrm{H}_{2}$, the "precursor" they define as the conduit to sooting. The second component is the formation of incipient particles in regions of high precursor concentration. Although something less than $10 \%$ of the total soot mass consists of these incipient particles, they are important because they provide the initial conditions for the subsequent growth phase. The second step, the "nucleation rate," is assumed proportional to the precursor concentration. $C_{\mathrm{C}, \mathrm{H}}$. The minimum particle size is taken to be $100 \mathrm{C}$ atoms. The third step is surface growth by adsorption. For their model they employ the results of previous experimental studies [17-19]. The fourth step is soot oxidation to $\mathrm{CO}$, which the authors assert occurs in a narrow region close to the flame on the oxidizer side. They also state that their "...predictions are comparatively insensitive to this reaction step." They employ $O$, as the oxidizer, though strong arguments have been made for $\mathrm{OH}$. The fifth component of their model is a particle agglomeration step. Interestingly, they employ detailed kinetic equations that have been derived for adiabatic flames: hence, their detailed mechanism should be applicable for lightly sooting flames. For the model equations only thermophoretic transport is specified for the soot, and a rather simple ad hoc correction was used to simulate the radiant heat loss from the flame: for the temperature distribution they wrote $T=T_{a d}$ (1 - $\left.C\left(T_{\mathrm{ad}} / T_{\max }\right)^{4}\right)$, where $T_{\mathrm{ad}}$ is the local temperature calculated adiabatically and $T_{\max }$ is the maximum value of $T_{3 d}$. Obviousty this corrertill cunnot change the arlalusciue snape of thr

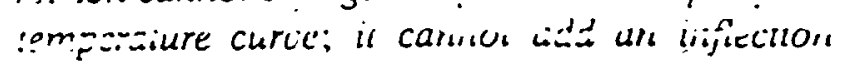
point in regions of high heat loss, for instance, as we have in Figs. $3 a$ and $3 b$. Nevertheless, $\Phi$ is fairly well predicted, with discrepancies attributed to "uncertainties in the gas-phase chemistry and the simplified treatment of the nonadiabaticity..." They conclude that for the 
strong temperature gradients in the particulate laver.

7. Athough our model is a highly simplified version of any available physical (experimental) counterpart. our discussion of the lirerature shows that many of our simplifications. restrictions and assumptions are consistent with empirical observations. Placing the "soot" layer adjacent to the reaction zone is in reasonable accord with the evidence. It is likely that (convection) $\gg$ (thermophoresis) in the studies we have reviewed, except for Ref. 12.

8. Arguments were presented supporting the need for a reliable and physically reasonable physicochemical mechanism for soot formation. The phenomenological approach, with its strong intuitive appeal, requires the existence of a convective flow. Although our zero-convective limit may not be realistic, it focuses attention on the deficiencies of phenomenological modeling.

9. An interesting set of equations (Eqs. 7.i, 7.ii) was obtained for describing the interaction of a DF and an enthalpy-loss region. When $H=H(\tau, Z)$, only these two equations need be examined. When the radiation term $d \bar{Q}_{R} / d Z$ also depends on the soot mass fraction, which itself depends at the very least on the fuel mass fraction and the temperature, we have a coupled system of three nonlinear ordinary differential equations. In the general case the situation is even more complicated, for the soot mass fraction depends on the "initial conditions" provided by the pyrolysis chemistry, the nucleation sequence, the surface growth rate, etc. As always, the number of coupled nonlinear equations increases.

The author gratefully acknowledges a stimulating discussion in the winter of 1991 with Dr. ivicnuei Deiichatsics, is : ic:i as the recetpl of an

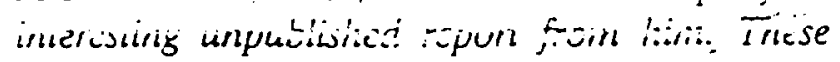
together suggested that theoretical models could indeed be developed to profitably examine this complex problem. This research was suppored by NASA under the microgravity combustion program, contract number NAG3-1271, monitored by $D r . K$ Sacksteder.

\section{REFERE:YCES}

1. Liñán. A.. Acra fistrona:u.. !:1007-1039 (107:)

¿. Williams. F. A. Comouston Theory, and ed.. Benjamin-Cummings. CA (1985).

¿. Tzeng. L.S.. Aireya. A.. and Wichman. I. S.. Combust. Flome 30:9: (1990).

4. Dagusé. T. Suuriani. A.. Darabiha. ... and Rolon. J. C.. IV Internationai Seminar on Flame siruciure. 18-22. August. Vocosioirsk. Russia. 1992.

5. Siegel. R.. and Howell. J. R.. Themal Radiation Heat Transfer. McGraw-Hill. Vew York, 1972.

6. Osizik. M. N.. Radialue Transjer and Interactions with Conduction and Concection. Wiley, NY. 1972.

7. Joulin. G.. and Deshaies. B.. Combust. Sci. Tecrinol. +7:299-\$15(1986).

8. Wagner. H. Gg., Secenteenth Symposium IIntema. rional) on Combustion. The Combustion Institute, Pittsburgh, (1979). pp. ;-19.

9. Kent, J. H., Jander. H.. and Wagner, H. Gy., Eigh. teenth Symposium (Intemational) on Combustion. The Combustion Instituce. Pitsburgh, (1981). pp. $1117-1126$

10. Symth. K. C., Miller. H. Dorfman. Mallard. W. G. and Santoro, R. H.. Combust. Flame 62:157-181 (1985).

11. Santoro. R. J.. Yeh. T. T., Horvath, J. J., and Semer. jian. H. G., Combust. Sci. Technol. 53:89-115 (1987).

12. Jackson. G. S., Avedisian. C. T., and Yang. J. C.. Int. J. Heat . Hass Trans. 35:2017-2033 (1992).

13. Kent. J. H., and Honnery, D. R., Combust. Flame 79:287-298 (1990).

14. Kennedy, 1. M.. Kollman. W., and Chen, J.YY.. Combust. Ficme 81:73-85 (1990).

15. Pagni. P. J.. and Okoh. C. I.. Twentieth Simposium (Intemarional) on Combustion. The Combustion Instituce. Pittsburgh. 198:. pp. 1045-1054.

16. Leung. K. M., Lindstedt. R. P., and Jones. W. P., Combust. Flame 87:289-305 (1991).

17. Harris. S. J., and Wiener. A. M., Combust. Sci. Technol. 31:155-167 (1983).

18. Harris. S. J., and Wiener. A. M., Combust. Sci. Technol. 32:267-275 (1983).

19. Vandsburger. U.. Kennedy, I. M.. and Glassman. I., Combust. Sci. Technol. 39:263-285 (1984).

20. Sohrab. S. H.. Liñan. A., and Williams, F. A.. Combust. Sci. Technol. 28:1+3-154 (1982).

21. Bonne, U., Combust. Flame 16:147-159 (1971).

22. Hottel, H., and Sarorim. A. F., Radiation Tranșter. McGraw-Hill. New York. 1967.

\section{APPENDIX}

Here we generalize the formulation of Sec. II vis à vis $H(Z)$. First, however, we reexamine the functional form we used there in slightly greater detail. We observe the following: (i) The derivative of $H(Z)$ is discontinuous at $Z_{R^{-}}$and $Z_{R^{+}}$. with values $\pm N_{R} \Delta Z_{R} / 2$ inside 
If $Y_{,} \gamma_{z Z}$ does not vanish. $H_{Z Z}$ is positive eterywhere on the interval $0<Z<1$. It is zero unly at the endpoints. Hence. $H$ topologically resembles Fig. 2b with $Z_{R^{-}}=0$ and $Z_{R^{-}}=1$. In reality. $Y_{V} / Y_{S R}$ will vanish at least on the side $Z<Z$. due to oxidation. If it vanishes abruptiy. as in Fig. 2b, no turther discussion is necessary: but if it vanishes smoothly, producing a "dog ear" near $Z=Z$; we may argue that the oxidization of the soot produces a local heat source. On the tuel side we do not expect any such heat sources: hence. either $Y_{\text {s }}$ vanishes abruptly or it remains non-zero to the wall. where the condition $T=T$, puts an end to the region $H_{z z}>0$ ). In either case. $H_{z z}$ should approach zero with a nonzero slope. This is qualitatively identical to what we have already done in Fig. 2.

Recelied 15 July 1993: recised 19 Junuary 1994 